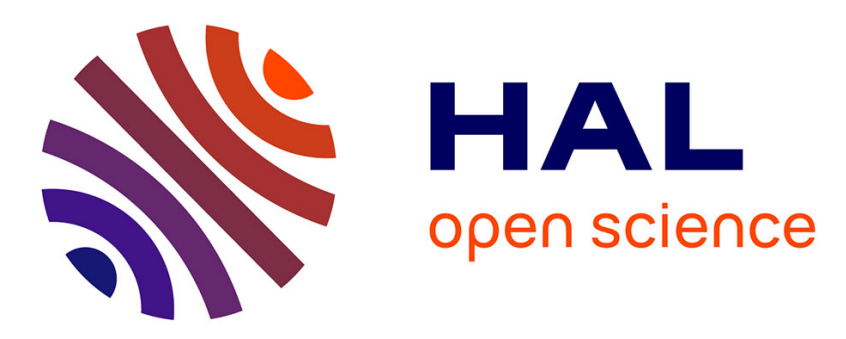

\title{
Electrically induced coalescence of two facing anchored water drops in oil
}

\author{
Jonathan Raisin, Pierre Atten, Frédéric Aitken, Jean-Luc Reboud
}

\section{To cite this version:}

Jonathan Raisin, Pierre Atten, Frédéric Aitken, Jean-Luc Reboud. Electrically induced coalescence of two facing anchored water drops in oil. International Conference on Dielectric Liquids (ICDL-2008), Jun 2008, Poitiers, France. hal-00366574

\section{HAL Id: hal-00366574 https://hal.science/hal-00366574}

Submitted on 31 Mar 2009

HAL is a multi-disciplinary open access archive for the deposit and dissemination of scientific research documents, whether they are published or not. The documents may come from teaching and research institutions in France or abroad, or from public or private research centers.
L'archive ouverte pluridisciplinaire HAL, est destinée au dépôt et à la diffusion de documents scientifiques de niveau recherche, publiés ou non, émanant des établissements d'enseignement et de recherche français ou étrangers, des laboratoires publics ou privés. 


\title{
Electrically Induced Coalescence of Two Facing Anchored Water Drops in Oil
}

\author{
J. Raisin, P. Atten, F. Aitken and J.-L. Reboud \\ G2Elab, CNRS, Univ. Joseph Fourier and INP Grenoble, \\ BP 166, 38042 Grenoble Cedex 9, France
}

\begin{abstract}
We investigate the deformation and coalescence of two closely spaced drops of conducting liquid suspended in an insulating fluid under the action of an electric field. The equations governing droplets deformation are derived in the case of drops subjected to a potential difference. The critical conditions for existence of a stationary solution are determined for drops anchored on capillary tubes in the asymptotic case of very close drops. In particular, electrocoalescence is predicted to occur when the interfaces distortions are such that the initial drops spacing has decreased by nearly $50 \%$. A numerical simulation gives the critical conditions for any spacing between the drops. Results compare favourably with the asymptotic approach in the common range of application.
\end{abstract}

\section{INTRODUCTION}

Electrocoalescence is the phenomenon of merging droplets of conducting liquid, which are suspended in an insulating liquid (or in a gas), under the action of an electric field.[1]. This phenomenon is important for the petroleum industry as it is used to increase the size of water droplets suspended in crude oils and, therefore, to drastically reduce the time required to separate water and oil phases under the gravity effect. Now, compact electrocoalescers that promote electrocoalescence in a very limited time, are often used [2]. But the control and increase of their efficiency are sometimes very difficult as the numerous processes involved in electrocoalescence are far from being fully understood. There is also another domain where this phenomenon of drops merging under the action of an electric field might be used: the lab-on-a-chip domain devoted to various chemical or biological analyses on very small amounts of products.

The action of an electric field on a water-in-oil emulsion is, first, to polarise the conducting water droplets and to promote an attraction between them. For not very close droplets, the dipole-dipole interaction force is very small and its effect can be neglected when the emulsion is in motion. For nearly contacting droplets pairs, conversely, the attraction force is strong; it induces a deformation of the droplets facing parts and often results in their merging. It is therefore necessary to distinguish the stage of build-up of drops pairs by the fluid motion (during which some droplets are brought in very close proximity), from the second stage, the coalescence itself, during which the interfaces deform and disrupt [3]. The determination of the probability of coalescence of two nearly colliding droplets as a function of the main parameters of the problem (electric field, drops size distribution, water volume fraction and flow properties) is a difficult problem. A pre-requisite is a good description of the interfaces disruption mechanism and the determination of the electro-coalescence critical conditions.

This problem of deformation and disruption of a water/oil interface has been studied recently in the particular configuration of a metallic sphere hanging above a horizontal layer of water [4] (this can be considered as the limit case of interaction between a very small and a big droplets). The major fact is that a static shape of the electrically stressed interface exists only for a limited deformation: the sphere-interface distance cannot decrease below about half of its value without field action [4]. General considerations [5] and an approximate treatment [6] extended the basic properties of instability conditions for two identical drops. However, these results are questionable as two free drops attracting each other move and the conditions of interface disruption are different from the ones characterising static (and immobile) drops.

We examine here the real static problem by considering drops anchored at the tip of capillary tubes (Fig. 1). We aim at determining the critical conditions of electrocoalescence between two conducting drops of same radius with a well defined potential difference.

\section{ASymptotic CASE Of Very Close Drops}

We consider two water drops of radius $R_{0}$ with a spacing $s_{0}$ suspended into an insulating fluid. The application of a potential difference $\Delta V$ between the drops induces an electric field $E$ and an electrostatic pressure $p_{e s}=\varepsilon E^{2} / 2$ at the interfaces ( $\varepsilon$ : permittivity of the suspending fluid). The problem is to determine the subsequent deformation of the drops and, in particular, of the facing zones of the interfaces.

At any point of a drop surface, the static equilibrium imposes that the pressure difference between the water $\left(p_{i n}\right)$ and the outer fluid $\left(p_{\text {out }}\right)$ is compensated for by the electrostatic pressure $p_{e s}$ and the capillary pressure $p_{c a p}$ associated with the surface tension (for small enough droplets the gravitational force is negligible - Bond number $B o<<1)$ :

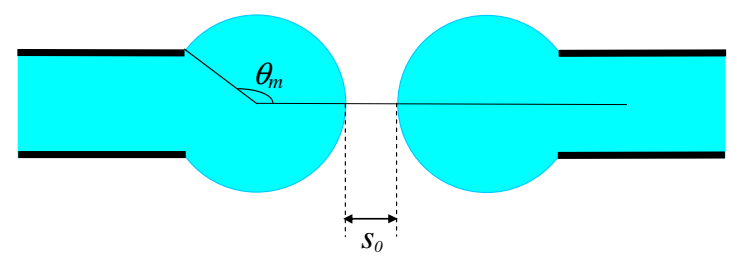

Fig. 1 - Drops anchored at the end of capillary tubes. 


$$
p_{\text {in }}-p_{\text {out }}=T\left(\frac{1}{R_{1}}+\frac{1}{R_{2}}\right)-\frac{1}{2} \varepsilon E^{2}
$$

here $R_{1}$ and $R_{2}$ are the principal radii of curvature and $T$ the surface tension. Instead of the cylindrical system of coordinates $r, \varphi, z$ considered in the classical problem of a drop deformed by the gravitational force [7], we use the spherical coordinates $r, \theta, \varphi$ (for one drop). By taking into account the axial symmetry of the problem, the equation for the interface of the considered drop is $r=g(\theta)$. Normalizing the spatial coordinates by the radius $R_{0}$ of non deformed drops and introducing the deformation $f$ scaled with the spacing $s_{0}$ between undistorted drops, we have (see Fig. 2) :

$$
g(\theta)=R_{0}[1+\sigma f(\theta)]
$$

where $\sigma=s_{0} / R_{0}$ is the relative initial spacing. The curvature of the surface is given by the divergence of the unit vector $\boldsymbol{n}$ normal to the interface. This leads to the expression :

$$
\frac{1}{R_{1}}+\frac{1}{R_{2}}=\frac{2}{\sqrt{g^{2}+g^{\prime 2}}}-\frac{\cos \theta}{\sin \theta} \frac{g^{\prime} / g}{\sqrt{g^{2}+g^{\prime 2}}}-\frac{g g^{\prime \prime}-g^{\prime 2}}{\left(g^{2}+g^{\prime 2}\right)^{3 / 2}}
$$

In the asymptotic case $\sigma<<1$, we approximate the field by $E=\Delta V / \Delta z$ where $\Delta z=s_{0}[1-2 f \cos \theta+(2 / \sigma)(1-\cos \theta)]$ is the distance between facing points of the interfaces. After introduction of non dimensional variables, we obtain the following equation governing the deformation profile $f\left(\delta p_{i n}\right.$ denotes the non dimensional change of the inner drop pressure under the field action) :

$$
\begin{array}{r}
(1+\sigma f) f^{\prime \prime}+\frac{\cos \theta}{\sin \theta} f^{\prime}\left[1+\sigma f+\frac{\sigma^{2} f^{\prime 2}}{1+\sigma f}\right]-\sigma^{\prime 2}= \\
-\frac{\left[(1+\sigma f)^{2}+\sigma^{2} f^{\prime 2}\right]^{3 / 2}}{\sigma}\left\{2-2 /\left[(1+\sigma)^{2}+\sigma^{2} f^{\prime 2}\right]^{1 / 2}\right. \\
\left.+\delta p_{\text {in }}+B e /\left[1-2 f \cos \theta+\frac{2}{\sigma}(1-\cos \theta)\right]^{2}\right\} \\
R_{0}+s_{0}
\end{array}
$$

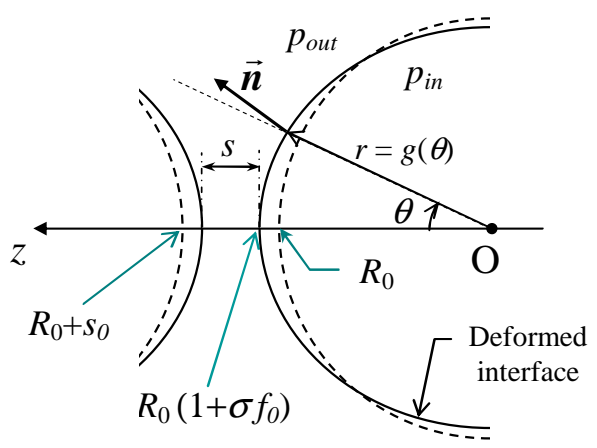

Fig. 2 - Distorted drop (full line : $r=g(\theta)=R_{0}+s_{0} f(\theta)$ ) and initial spherical drop (dashed line) in spherical coordinates.

The boundary conditions are simple and concern:
i) the derivative of $f$ on the axis :
$f^{\prime}=0$ at $\theta=0$
ii) the anchoring of the drops :
$f=0$ at $\theta=\theta_{m}$

iii) conservation of drop volume $\mathrm{Vol}: \quad \delta(\mathrm{Vol})=0$

Solutions of eq. (4) have been obtained by numerical integration using the embedded Runge-Kutta-Fehlberg method. Determining solutions is not easy because a double shooting method is required to solve the problem: the value $f_{0}$ of the deformation on the axis $(\theta=0)$ being given, we have to determine the appropriate values of $B e$ and $\delta p_{\text {in }}$ that satisfy the boundary conditions and the drop volume conservation.

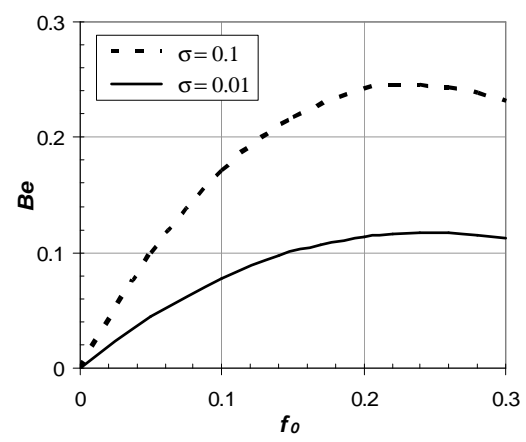

Fig. 3 - Variations of the electrical Bond number $B e$ as a function of the normalized deformation $f_{0}$ on the axis for hemispherical drops $\left(\theta_{m}=\pi / 2\right)$.

Fig. 3 illustrates the relation between the electrical Bond number $B e$ and the normalized deformation $f_{0}$ at the axis in the case of hemispherical menisci $\left(\theta_{m}=\pi / 2\right)$. The approximately parabolic shape of the $\operatorname{Be}\left(f_{0}\right)$ curves is quite analogous to the one obtained by the previous approximate treatments [6]. The maximum which corresponds to the critical conditions is reached for $f_{0}$ between 0.24 and 0.25 (Fig. 3); this leads to $s_{c r i t}$ only slightly higher than $0.5 s_{0}$.

The distribution of the interface deformation $f(\theta)$ is shown in Fig. 4 for different $B e$ values again for hemispherical drops $\left(\theta_{m}=\pi / 2\right)$. Clearly there is an elongation of the drops which is only slightly dependent on the ratio $\sigma$ of initial spacing $s_{0}$ and radius $R_{0}$ [8]. The critical conditions vary in a rather limited way on $\sigma$ for all values of the angle $\theta_{m}$ (Fig. 5).

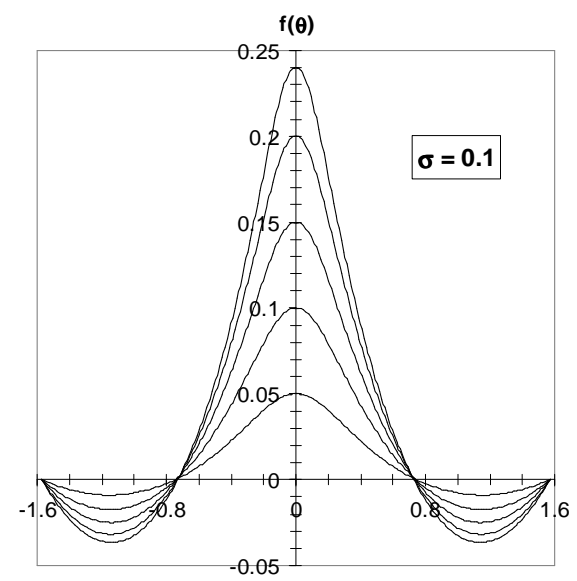

Fig. 4 - Deformation $f$ of the interface as a function of the angle $\theta$ (in radians) for different $B e$ numbers in the case of a hemispherical meniscus $\left(\theta_{m}=\pi / 2\right)$ 


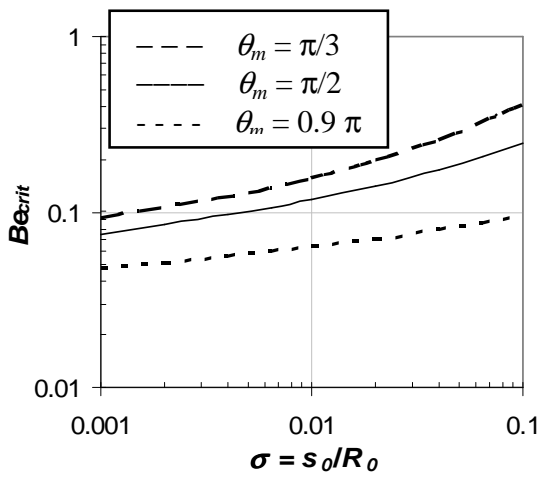

Fig. 5 - Critical value of the electric Bond number $B e_{c r i t}$ as a function of the relative initial spacing $\sigma$ for 3 values of the angle $\theta_{m}$ characterizing the meniscus.

\section{CRitical Conditions In The General CASE}

When the spacing $s_{0}$ between the interfaces of the two anchored drops is not very small compared with their radius $R_{0}$, the approximate expression retained in $\$ 2$ for the electric field is not valid. It is then necessary to solve the Laplace equation for the electric potential in the domain outside the deformed drops (and the capillary tube). This has been performed using the commercial software Comsol Multiphysics ${ }^{\mathrm{TM}}$.

The time dependent Navier-Stokes equations are solved in the water (NS1) and oil (NS2) media (Fig. 6). Because the two fluids are incompressible we have volume conservation of the droplets. These fluid dynamic equations are coupled with the successive solution of the Laplace equation applied to the oil medium, water being considered as perfectly conductive, thus at constant electric potential. The deformation of the water-oil interface is implemented by using an Arbitrary LagrangianEulerian formulation with deforming meshes. This approach allows to define the forces that act on the interface, such as surface tension and electrostatic pressure, and track the interface deformation in a very accurate way. In order to obtain static solutions for the deformed drops, the applied voltage is varied very slowly in time so that the inertial and viscous terms remain in practice negligibly small. All the test cases were computed considering axial symmetry, and, in that particular case of two droplets of same radius, using the plane of symmetry between the drops.

Main modification of the standard software concerns the computation of the surface tension forces performed through a weak formulation of the fluid dynamics equation [9] adapted to the axial symmetry conditions. At each time step, the electric field $\mathrm{E}$ is computed in the oil domain, taking into account the actual surface deformation of the water drop. Boundary conditions imposed for Navier Stokes equations in the two liquid domains are:

i) Constant static pressure is imposed at the upper boundary of the oil domain. The pressure computed in oil, modified by electrostatic pressure $-\varepsilon E^{2} / 2$, is applied as boundary condition at the interface for the water domain. Surface tension $\mathrm{T}$ and local curvature involved in equation (1) are implemented indirectly through the weak formulation. ii) The velocity field computed within the water droplet, ensuring the volume conservation, is in its turn applied as boundary condition at the interface for the oil domain.

iii) Liquid velocity at the water-oil interface is applied in a Lagrangian way to move the boundary. The water-oil interface is closed, above the anchorage point, by a vertical impervious fixed boundary.

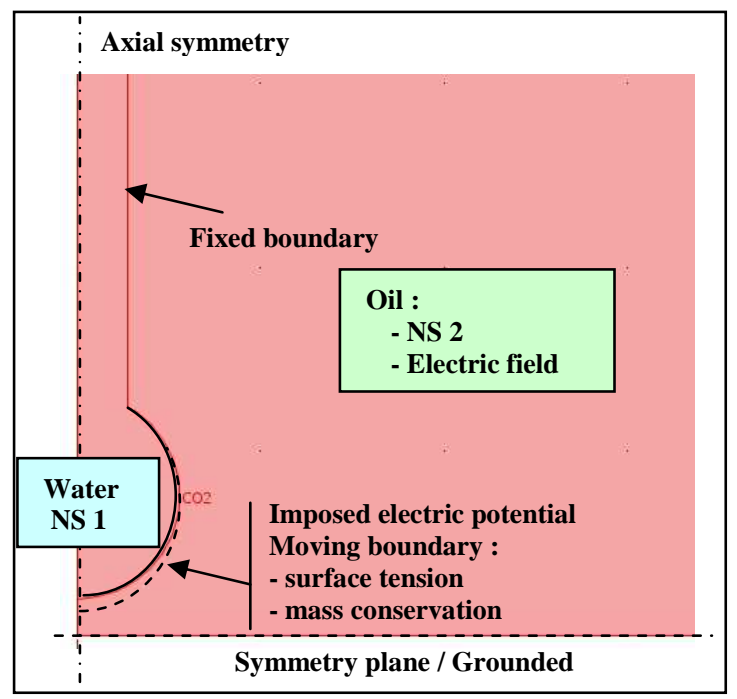

Fig. 6 - Geometry and equations solved

Fig. 7 illustrates the instantaneous shape of a deforming droplet and associated fields, in conditions very close to the critical potential difference $\Delta V_{\text {crit }}$. The transient calculation was performed with a slow rise of the voltage: $\Delta V / \Delta t=0.1$ $\mathrm{V} / \mathrm{s}$. Initial non dimensional spacing $\sigma=s_{0} / R_{0}=0.7$ is here much higher than the limit allowing the asymptotic case formulation, with its simple electric field approximation. Electric field lines are drawn in the oil domain to illustrate the point. A small pressure gradient can be seen inside the water, due to increasing electrostatic pressure on the interface and associated with the very close appearance of the surface instability.

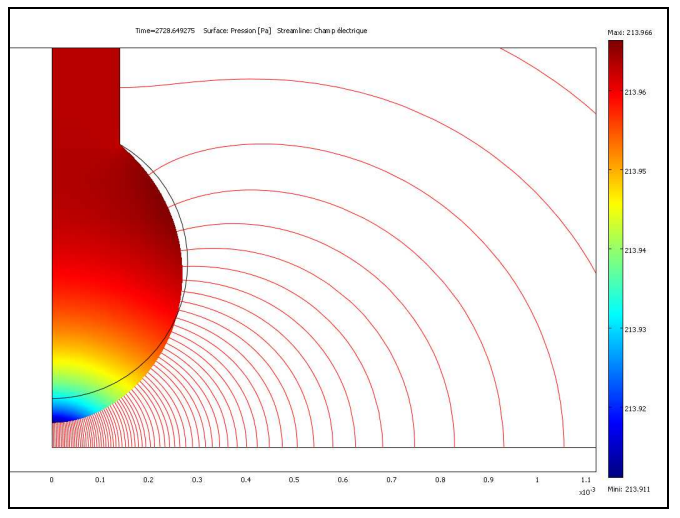

Fig. 7 - Deformation of droplet shape near the critical conditions, electric streamlines in the outer field (oil), pressure field in the water (full color scale: $0.05 \mathrm{~Pa}$ ). $\theta_{m}=150^{\circ}, R_{o}=0.28 \mathrm{~mm}, \sigma=0.7$, $T=25 \mathrm{mN} / \mathrm{m}, \varepsilon=21.2 \mathrm{pF} / \mathrm{m}$. 


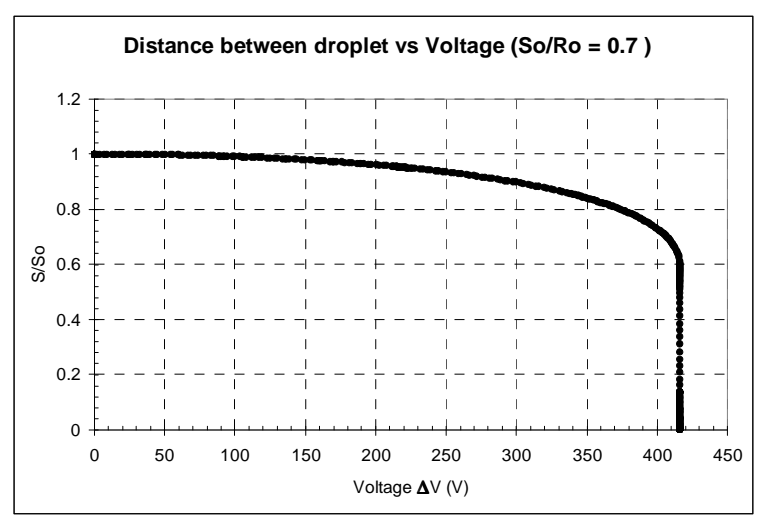

Fig. 8 - Time-dependent spacing $s$ versus increasing voltage $\Delta V . \theta_{m}=\pi / 2$, $R_{o}=0.28 \mathrm{~mm}, \sigma=0.7, T=25 \mathrm{mN} / \mathrm{m}, \varepsilon=21.2 \mathrm{pF} / \mathrm{m}$, $B e_{\text {crit }}=(\varepsilon / 2)\left(\Delta V^{2} / s_{0}^{2}\right)\left(R_{0} / T\right)=0.512$.

Spacing $s$ is drawn on Fig. 8 as a function of potential difference $\Delta V$. It can be seen that the appearance of the interfacial instability corresponds to a very sharp drop of the curve that provides an accurate evaluation of $\Delta V_{\text {crit }}$. The critical spacing $s_{c r i t}$ appears to be slightly larger than $0.6 s_{0}$. The difference with the value close to $0.5 s_{0}$, obtained previously by the asymptotic approach, can be mainly attributed to the effects of large deformation due to the larger initial spacing.

Results of the asymptotic and numerical approaches are plotted on Fig. 9 as a function of the relative spacing $\sigma$. In the range $0.01-0.1$ for $\sigma$, there is a good agreement between the two models, validating the numerical approach proposed here.

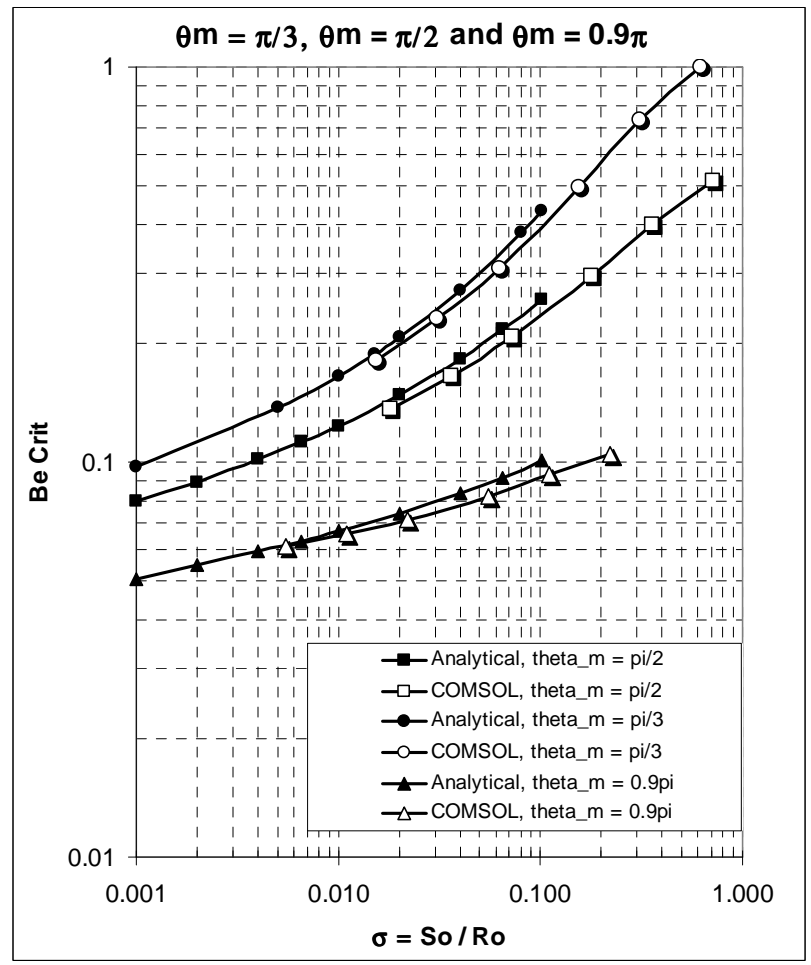

Fig. 9 - Critical value of the electric Bond number $B e_{\text {crit }}$ as a function of the relative initial spacing $\sigma$. Asymptotic and numerical approaches.
As expected the difference between results given by the two methods increases with $\sigma$. The general numerical method gives critical values slightly lower than the asymptotic ones because the real electric field on the interface is slightly higher than the uniform field retained in the asymptotic approach, thus requiring a slightly lower applied voltage. Finally let us note that the influence of relative spacing $\sigma$ on the critical condition becomes stronger when $\sigma$ is increased.

\section{CONCLUSION}

Two different methods have been compared to evaluate the critical conditions leading to the electrocoalescence of close anchored water droplets in oil. Asymptotic approach is applied in the range of small relative spacing, while numerical simulation was developed to extend the results when the initial spacing becomes of the same order of magnitude than the drop radius. The two methods show a good agreement in their common range of application. Further investigations with the numerical model are planed to extend the analyses to transient electric fields and associated coalescence criteria.

\section{ACKNOWLEDGMENT}

This work is developed in the framework of a collaborative research between the French CNRS and the Consortium working on the project "Electrocoalescence - Criteria for an efficient process in real crude oil systems"; co-ordinated by SINTEF Energy Research. Contact person is L.E. Lundgaard. The project is supported by The Research Council of Norway, under the contract no: 169466/S30, and by the following industrial partners: Vetco Aibel AS, Aker Kvaerner Process Systems AS, StatoilHydro ASA, BP Exploration Operating Company Ltd, Shell Technology Norway AS, Petrobras.

\section{REFERENCES}

[1] L.E. Lundgaard, G. Berg, S. Ingebrigtsen, P. Atten, "Electrocoalescence for oil-water separation: Fundamental aspects", in Emulsions and emulsion stability, J. Sjöblom, Ed., Surfactant science series, vol. 132, Taylor \& Francis, 2006, pp. 549-592.

[2] O. Urdahl, N.J. Wayth, H. Fordedal, T.J. Williams, A.G. Bailey, "Compact electrostatic coalescer technology" in Encuclopedic handbook of emulsion technology, J. Sjöblom, Ed., Marcel Dekker, 2001, pp. 679694.

[3] P. Atten, "Electrocoalescence of water droplets in an insulating liquid", $J$. Electrostatics, 30, pp. 259-269, 1993.

[4] P. Atten, F. Aitken, D. Koulova-Nenova, "Field-induced deformation and disruption of a planar water-oil interface under the influence of a conducting sphere", Proceed. ICDL 2005, IEEE-Cat.-No. 05CH37643, pp. 165-168, 2005.

[5] P. Atten, L. Lundgaard, G. Berg, "A simplified model of electrocoalescence of two close droplets in oil", J. Electrostatics, 64, pp. 550554, 2006.

[6] P. Atten, "Critical conditions for electrically induced coalescence of two very close water droplets in oil", Proceed. ICDL 2005, IEEE-Cat.-No. 05CH37643, pp. 177-180, 2005.

[7] F. Bashforth and J.C. Adams, "An attempt to test the theories of capillary action", London \& Cambridge University Press, 1883.

[8] P. Atten, F. Aitken, "Electrocoalescence criterion for two close water drops", Conf. Record 2007 IEEE-IAS 42 $2^{\text {nd }}$-IAS-Annual-Meeting, 5 pages (CD), 2007.

[9] P.H. Saksono, D. Peric, "On finite element modeling of surface tension", Comp. Mech., 38, pp. 265-281, 2006. 